\title{
Note on Conditional Constructivity
}

\author{
Matthias Baaz \\ Institute of Discrete Mathematics and Geometry, Technical University of Vienna, \\ Wiedner Hauptstrasse 8-10 / E104, A-1040 Vienna, Austria \\ baaz@logic.at
}

\begin{abstract}
In this note we provide a straightforward translation $\mathcal{C}_{p}^{\Gamma}(T)$ for sets of formulas $T$ and $H_{\Gamma}(\exists x A(x))$ for existential formulas $\exists x A(x)$ s.t. $\mathcal{C}_{p}^{\Gamma}(T) \vdash H_{\Gamma}(\exists x A(x))$ expresses " $\exists x A(x)$ is derivable constructively from $T$ iff it is derivable at all".
\end{abstract}

\section{Preliminaries}

The strength of mathematical logic lies often in its ability to express metamathematical statements on a mathematical level. In this note we deal with conditional constructive provability, i.e. with statements "if $\exists x A(x)$ is provable at all from $T$ then it is constructively provable". In addition, the signature of the witness can be specified arbitrarily.

The idea is to add a variable position $x$ to every atomic formula, let $B^{*}(x)$ be the transform of $B$ and let $A^{*}(y, x)$ be the transform of $\exists y A(y)$ after deletion of the outermost existential quantifier. Let $T^{*}$ be the $\forall$-closure of $\left\{B^{*}(x) \mid B\right.$ in $\left.T\right\}$. Then $T^{*} \vdash \exists x \forall y\left(A^{*}(y, x) \supset A^{*}(x, x)\right)$ expresses the desired statement.

The reasons to express constructive provability and conditional constructive provability using a translation within classical logic are mainly the following:

- It is not possible to characterize classical constructive provability by a socalled constructive logic $\mathcal{L}$ weaker than classical logic: There will be always $T$ and $\exists x A(x)$, such that $T \vdash \exists x A(x)$ non constructively in $\mathcal{L}$ but $T \vdash A(t)$ for some $t$ in classical logic 1

- Usual classical models can be used to study classical non-provability in the constructive sense in the presence of provability.

- " $\exists x A(x)$ is constructively provable" can be used as assumption/axiom without extending the framework of classical logic, in case of conditional constructive provability as meaningful assumption/axiom for all extensions of a given theory.

\section{$2 \quad \mathcal{C}_{p}^{\Gamma} / \mathcal{C}_{c}^{\Gamma}$}

Let $\vdash$ denote classical deduction and let $\operatorname{cl}_{\exists}(A)\left(\operatorname{cl}_{\forall}(A)\right)$ be the existential (universal) closure of $A$.

${ }^{1} T \equiv((Q \vee \neg Q) \supset P(0)) \wedge(P(0) \vee P(1))$ and $\exists x P(x)$ provide an example for any intermediate first-order logic $\mathcal{L}$. 
Let $T$ be a theory, $\Gamma$ be assigned to $A \equiv \exists y A^{\prime}(y)$, and assume that all bound variables are different to $x 2$

Define

$$
\begin{aligned}
& \psi_{\Gamma}^{x}(z) \equiv z \quad(z \text { a bound or free variable }) \\
& \psi_{\Gamma}^{x}(c) \equiv c^{*}(x) \\
& \left(c \text { constant, } c \notin \Gamma, c^{*} \text { is a new function symbol of arity } 1\right) \\
& \psi_{\Gamma}^{x}(c) \equiv c \quad(c \text { constant, } c \in \Gamma) \\
& \psi_{\Gamma}^{x}\left(f\left(t_{1}, \ldots, t_{r}\right)\right) \equiv f^{*}\left(\psi_{\Gamma}^{x}\left(t_{1}\right), \ldots, \psi_{\Gamma}^{x}\left(t_{r}\right), x\right) \\
& \left(f \notin \Gamma, f^{*} \text { a new function symbol of arity } r+1\right) \\
& \psi_{\Gamma}^{x}\left(f\left(t_{1}, \ldots, t_{r}\right)\right) \equiv f\left(\psi_{\Gamma}^{x}\left(t_{1}\right), \ldots, \psi_{\Gamma}^{x}\left(t_{r}\right)\right) \quad(f \in \Gamma) \\
& \psi_{\Gamma}^{x}\left(P\left(v_{1}, \ldots, v_{n}\right)\right) \equiv P^{*}\left(\psi_{\Gamma}^{x}\left(v_{1}\right), \ldots, \psi_{\Gamma}^{x}\left(v_{n}\right), x\right) \\
& \left(P^{*} \mathrm{a} \text { new predicate symbol of arity } n+1\right) \\
& \psi_{\Gamma}^{x}(\neg A) \equiv \neg \psi_{\Gamma}^{x}(A) \\
& \psi_{\Gamma}^{x}(A \vee B) \equiv \psi_{\Gamma}^{x}(A) \vee \psi_{\Gamma}^{x}(B) \\
& \psi_{\Gamma}^{x}(A \wedge B) \equiv \psi_{\Gamma}^{x}(A) \wedge \psi_{\Gamma}^{x}(B) \\
& \psi_{\Gamma}^{x}(A \supset B) \equiv \psi_{\Gamma}^{x}(A) \supset \psi_{\Gamma}^{x}(B) \\
& \psi_{\Gamma}^{x}(\exists y A) \equiv \exists y \psi_{\Gamma}^{x}(A) \\
& \psi_{\Gamma}^{x}(\forall y A) \equiv \forall y \psi_{\Gamma}^{x}(A) . \\
& \mathcal{C}_{p}^{\Gamma}(T) \equiv\left\{\mathrm{cl}_{\forall}\left(\psi_{\Gamma}^{x}(B)\right) \mid B \in T\right\} \\
& \mathcal{C}_{c}^{\Gamma}(\exists y A(y)) \equiv \exists x \psi_{\Gamma}^{x}(A(x)) \bigsqcup^{3}
\end{aligned}
$$

Example 1. Consider $P(0) \vee P(1)$ and $\exists y P(y)$.

$$
\begin{aligned}
& \Gamma=\{0,1\} \\
& \psi_{\Gamma}^{x}(P(0) \vee P(1)) \equiv P^{*}(0, x) \vee P^{*}(1, x) \quad \psi_{\Gamma}^{x}(\exists y P(y)) \equiv \exists y P^{*}(y, x) \\
& \mathcal{C}_{p}^{\Gamma}(P(0) \vee P(1)) \equiv \forall x\left(P^{*}(0, x) \vee P^{*}(1, x)\right) \\
& \mathcal{C}_{c}^{\Gamma}(\exists y P(y)) \equiv \exists x P^{*}(x, x) \\
& \Gamma=\{1\} \\
& \psi_{\Gamma}^{x}(P(0) \vee P(1)) \equiv P^{*}\left(0^{*}(x), x\right) \vee P^{*}(1, x) \quad \psi_{\Gamma}^{x}(\exists y P(y)) \equiv \exists y P^{*}(y, x) \\
& \mathcal{C}_{p}^{\Gamma}(P(0) \vee P(1)) \equiv \forall x\left(P^{*}\left(0^{*}(x), x\right) \vee P^{*}(1, x)\right) \\
& \mathcal{C}_{c}^{\Gamma}(\exists y P(y)) \equiv \exists x P^{*}(x, x)
\end{aligned}
$$

${ }^{2} \Gamma$ is a signature for the specification of terms which are admitted as witnesses.

${ }^{3}$ Note that by duality the translation $\mathcal{C}_{p}^{\Gamma} / \mathcal{C}_{c}^{\Gamma}$ can be used to control resources, i.e. to a priori limit the number of instances of universal axioms to be used in the proof. 
Theorem 1. $T \vdash A(t)$ for some closed term of the signature of $\Gamma$ iff $\mathcal{C}_{p}^{\Gamma}(T) \vdash$ $\mathcal{C}_{c}^{\Gamma}(\exists x A(x))$.

Proof. See [1] or 2], for a more general setting $\left(\mathcal{C}_{p}^{\Gamma} / \mathcal{C}_{c}^{\Gamma}\right.$ correspond to $\mathcal{C}_{p}^{\chi} / \mathcal{C}_{c}^{\chi}$ with $\left.\chi=\left\langle\begin{array}{l}1 \\ \Gamma\end{array}\right\rangle\right)$. The proof uses the following property of resolution refutations. For sets of clauses $\left\{(\neg) L_{i 1}\left(\bar{y}_{i 1}, x\right), \ldots,(\neg) L_{i k_{i}}\left(\bar{y}_{i k_{i}}, x\right)\right\}$ ground substitutions of refutations must always coincide at the $x$-position: $x$ stores the term $t$ making the argument constructive. Function symbols not in $\Gamma$ cannot occur in $t$ as their translation depends on $x$.

Example 2. $P(0) \vee P(1)$ does not prove $\exists x P(x)$ constructively as

$$
\begin{aligned}
& \mathcal{C}_{p}^{\{0,1\}}(P(0) \vee P(1)) \equiv \forall x\left(P^{*}(0, x) \vee P^{*}(1, x)\right), \\
& \mathcal{C}_{c}^{\{0,1\}}(\exists x P(x)) \equiv \exists x P^{*}(x, x), \\
& \text { and } \forall x\left(P^{*}(0, x) \vee P^{*}(1, x)\right) \forall \exists x P^{*}(x, x) .
\end{aligned}
$$

Example 3. $P(0), P(0) \vee P(1)$ do prove $\exists x P(x)$ constructively. This is not the case if the signature is restricted to 1 as

$$
\begin{aligned}
& \mathcal{C}_{p}^{\{1\}}(P(0) \vee P(1)) \equiv \forall x\left(P^{*}\left(0^{x}(x), x\right) \vee P^{*}(1, x)\right), \\
& \mathcal{C}_{p}^{\{1\}}(P(0)) \equiv \forall x P^{*}\left(0^{x}(x), x\right), \\
& \mathcal{C}_{c}^{\{1\}}(\exists x P(x)) \equiv \exists x P^{*}(x, x), \\
& \text { and } \forall x\left(P^{*}\left(0^{x}(x), x\right)\right), \forall x\left(P^{*}\left(0^{x}(x), x\right) \vee P^{*}(1, x)\right) \forall \exists x P^{*}(x, x) .
\end{aligned}
$$

Example 4. $\exists x P(x)$ does not prove $\exists x P(x)$ constructively $(\Gamma=\{0\})$ as

$$
\begin{aligned}
& \mathcal{C}_{c}^{\{0\}}(\exists x P(x)) \equiv \forall x \exists y P^{*}(x, y), \\
& \mathcal{C}_{c}^{\{1\}}(\exists x P(x)) \equiv \exists x P^{*}(x, x), \\
& \text { and } \forall x \exists y P^{*}(x, y) \nvdash \exists x P^{*}(x, x) .
\end{aligned}
$$

Example 5. We use a well known example of non-constructivity (cf. 3]). There are irrational numbers $a, b$ such that $a^{b}$ is rational: consider $\sqrt{2}^{\sqrt{2}}$. If $\sqrt{2}^{\sqrt{2}}$ is rational let $a=b=\sqrt{2}$. If $\sqrt{2}^{\sqrt{2}}$ is irrational let $a=\sqrt{2}^{\sqrt{2}}$ and $b=\sqrt{2}$, where

$$
a^{b}=\left(\sqrt{2}^{\sqrt{2}}\right)^{\sqrt{2}}=\sqrt{2}^{2}=2 .
$$

We formalize this argument using the predicate $R(x)$, the constant $\sqrt{2}$, and the function $\exp (x, y)$ (also written as $\left.x^{y}\right)$.

$$
\text { Let } \begin{gathered}
T=\neg R(\sqrt{2}), R\left(\left(\sqrt{2}^{\sqrt{2}}\right)^{\sqrt{2}}\right) \text { and } \Gamma=\{\sqrt{2}, \exp (x, y)\} . \\
T \vdash \exists x \exists y\left(\neg R(x) \wedge \neg R(y) \wedge R\left(x^{y}\right)\right)
\end{gathered}
$$




$$
\mathcal{C}_{p}^{\Gamma}(T)=\forall x \neg R^{*}(\sqrt{2}, x), \forall x R^{*}\left(\left(\sqrt{2}^{\sqrt{2}}\right)^{\sqrt{2}}, x\right)
$$

$\mathcal{C}_{c}^{\Gamma}\left(\exists x \exists y\left(\neg R(x) \wedge \neg R(y) \wedge R\left(x^{y}\right)\right)\right)=\exists x \exists y\left(\neg R^{*}(x, x) \wedge \neg R^{*}(y, x) \wedge R^{*}\left(x^{y}, x\right)\right)$.

The following structure $\left\langle M, \bar{R}^{*}, \exp , \sqrt{2}\right\rangle$ is a model for

$$
\forall x \neg R^{*}(\sqrt{2}, x), \forall x R^{*}\left(\left(\sqrt{2}^{\sqrt{2}}\right)^{\sqrt{2}}, x\right), \forall x \forall y\left(R^{*}(x, x) \vee R^{*}(y, x) \vee \neg R^{*}\left(x^{y}, x\right)\right)
$$

and consequently a counterexample to

$$
\mathcal{C}_{p}^{\Gamma}(T) \vdash \mathcal{C}_{c}^{\Gamma}\left(\exists x \exists y\left(\neg R^{*}(x, x) \wedge \neg R^{*}(y, x) \wedge R^{*}\left(x^{y}, x\right)\right)\right) .
$$

$M$ is the set of terms constructed from $\sqrt{2}$ and $\exp (x, y)$,

$(\sqrt{2}, v) \notin \bar{R}^{*}$ for all $v$,

$\left(\sqrt{2}^{\sqrt{2}}, \sqrt{2}\right) \notin \bar{R}^{*}$,

$(u, v) \in \bar{R}^{*}$ otherwise.

Therefore there is no term $t$ such that

$$
T \vdash \exists y\left(\neg R(t) \wedge \neg R(y) \wedge R\left(t^{y}\right)\right) .
$$

\section{Conditional Constructivity}

Let $H_{\Gamma}(\exists y A(y)) \equiv \exists x \forall y\left(\psi_{\Gamma}^{x}(A(y)) \supset \psi_{\Gamma}^{x}(A(x))\right)$.

Example 6. $H_{\Gamma}(\exists x P(x)) \equiv \exists x \forall y\left(P^{*}(y, x) \supset P^{*}(x, x)\right)$.

Proposition 1. $T \vdash A \Leftrightarrow\left\{\forall x \psi_{\Gamma}^{x}(B) \mid B \in T\right\} \vdash \forall x \psi_{\Gamma}^{x}(A)$.

Proof. By induction on the proof length.

\section{Theorem 2.}

(i) $T \vdash A(t)$ for some closed term of the signature of $\Gamma \Rightarrow \mathcal{C}_{p}^{\Gamma}(T) \vdash H_{\Gamma}(\exists x$ $A(x))$.

(ii) $\mathcal{C}_{p}^{\Gamma}(T) \vdash H_{\Gamma}(\exists x A(x)), T \subseteq S, S \vdash \exists x A(x) \Rightarrow S \vdash A(t)$ for some closed term of the signature of $\Gamma$.

Proof.

(i) $T \vdash A(t)$ for some closed term of the signature of $\Gamma \Rightarrow \mathcal{C}_{p}^{\Gamma}(T) \vdash \exists x \psi_{\Gamma}^{x}(A(x))$ by Theorem 1. $\Rightarrow \mathcal{C}_{p}^{\Gamma}(T) \vdash \exists x \forall y\left(\psi_{\Gamma}^{x}(A(y)) \supset \psi_{\Gamma}^{x}(A(x))\right) \equiv H_{\Gamma}(\exists y A(y))$.

(ii) $\mathcal{C}_{p}^{\Gamma}(T) \vdash H_{\Gamma}(\exists y A(y)), T \subseteq S, S \vdash \exists x A(x) \Rightarrow \mathcal{C}_{p}^{\Gamma}(S) \vdash H_{\Gamma}(\exists y A(y))$, $\mathcal{C}_{p}^{\Gamma}(S) \vdash \forall x \exists y \psi_{\Gamma}^{x}(A(y)) \Rightarrow \mathcal{C}_{p}^{\Gamma}(S) \vdash \exists x \psi_{\Gamma}^{x}(A(x))$ as

$\vdash H_{\Gamma}(\exists y A(y)) \supset\left(\forall x \exists y \psi_{\Gamma}^{x}(A(y))\right) \supset \exists x \psi_{\Gamma}^{x}(A(x))$ and $\mathcal{C}_{p}^{\Gamma}(S) \vdash \forall x \exists y$ $\psi_{\Gamma}^{x}(A(y))$ by Proposition $1 \Rightarrow S \vdash A(t)$ for some closed term $t$ in the signature $\Gamma$ by Theorem 1 .

Note that $t$ is already "known" to $T$ as $H_{\Gamma}(\exists y A(y)) \equiv \mathcal{C}_{c}^{\Gamma}(\exists x \forall y(A(y) \supset$ $A(x)))(\vdash \exists x \forall y(A(y) \supset A(x)) !)$. 
Example 7. $P(0) \vee P(1), \exists x P(x) \supset P(0)$ prove the conditional constructivity for $\exists y P(y)$, prove $\exists x P(x)$, but do not prove $\exists x P(x)$ constructively.

$$
\begin{aligned}
& \mathcal{C}_{p}^{\{0,1\}}(P(0) \vee P(1)) \equiv \forall x\left(P^{*}(0, x) \vee P^{*}(1, x)\right) \\
& \mathcal{C}_{p}^{\{0,1\}}(\exists x P(x) \supset P(0)) \equiv \forall x\left(\exists y P^{*}(y, x) \supset P^{*}(0, x)\right) \\
& H_{\{0,1\}}(\exists y P(y)) \equiv \exists x \forall y\left(P^{*}(y, x) \supset P^{*}(x, x)\right) .
\end{aligned}
$$

Obviously

$$
\mathcal{C}_{p}^{\{0,1\}}(P(0) \vee P(1)), \mathcal{C}_{p}^{\{0,1\}}(\exists x P(x) \supset P(0)) \vdash H_{\{0,1\}}(\exists y P(y)) .
$$

\section{Conclusion}

The establishment of conditional (relative) constructivity is an essential feature of constructive mathematics, as relative constructivity proofs allow for the combination of constructive parts of non-constructive proofs with suitable constructive specializations. The translation presented in this paper makes it possible to use classical models to analyze, why relative constructivity fails.

\section{References}

1. Baaz, M.: Note on a translation to characterize constructivity. J. Proc. Steklov Inst. Math. 242, 125-129 (2003)

2. Baaz, M.: Controlling witnesses. Annals of Pure and Applied Logic 136, 22-29 (2005)

3. Troelstra, A., van Dalen, D.: Constructivism in Mathematics, An Introduction, vol. 1. North-Holland, Amsterdam (1988) 